\title{
Interdigitated Extended Gate Field Effect Transistor Without Reference Electrode
}

\author{
GHUSOON M. ALI ${ }^{1,2,3}$ \\ 1.-Electrical Engineering Department, College of Engineering, Al-Mustansiriyah University, \\ Baghdad, Iraq. 2.—e-mail: ghusoon.ali@gmail.com. 3.—e-mail: ghusoon_m_a@yahoo.com
}

An interdigitated extended gate field effect transistor (IEGFET) has been proposed as a modified $\mathrm{pH}$ sensor structure of an extended gate field effect transistor (EGFET). The reference electrode and the extended gate in the conventional device have been replaced by a single interdigitated extended gate. A metal-semiconductor-metal interdigitated extended gate containing two multi-finger $\mathrm{Ni}$ electrodes based on zinc oxide $(\mathrm{ZnO})$ thin film as a $\mathrm{pH}$ sensitive membrane. $\mathrm{ZnO}$ thin film was grown on a $p$-type $\mathrm{Si}$ (100) substrate by the sol-gel technique. The fabricated extended gate is connected to a commercial metal-oxide-semiconductor field-effect transistor device in CD4007UB. The experimental data show that this structure has real time and linear $\mathrm{pH}$ voltage and current sensitivities in a concentration range between $\mathrm{pH} 4$ and 11. The voltage and current sensitivities are found to be about $22.4 \mathrm{mV} / \mathrm{pH}$ and $45 \mu \mathrm{A} / \mathrm{pH}$, respectively. Reference electrode elimination makes the IEGFET device simple to fabricate, easy to carry out the measurements, needing a small volume of solution to test and suitable for disposable biosensor applications. Furthermore, this uncomplicated structure could be extended to fabricate multiple ions microsensors and lab-on-chip devices.

Key words: Interdigitated, EGFET, ZnO, thin-film, pH-sensors, without-reference-electrode

\section{INTRODUCTION}

A thin film oxide semiconductor as a sensitive layer combined with a field effect transistor (FET) is one of the most attractive techniques for $\mathrm{pH}$ sensor development. ${ }^{1}$ This method has drawn significant interest by providing many important advantages such as flexibility to miniaturization, cost-effectiveness, disposability, and real-time measurement. ${ }^{2}$ An ion-sensitive field effect transistor (ISFET) was first proposed by Bergveld in the $1970 \mathrm{~s}^{3}$ An ISFET was employed to replace the fragile glass electrode in $\mathrm{pH}$ and ion concentration measurements. ${ }^{4,5}$ However, the device suffered from poor isolation between the device and the solution causing the harsh

(Received June 25, 2016; accepted October 7, 2016; published online October 21, 2016) environment in the solutions to degrade the device's reliability. ${ }^{6,7}$ To overcome the disadvantages, in 1983, Van der Spiegel et al. ${ }^{8}$ improved an ISFET structure as an extended gate field effect transistor (EGFET). Later, in 2000, Chi et al. ${ }^{9}$ presented an EGFET with a commercial metal-oxide-semiconductor field-effect transistor (MOSFET). This arrangement has better long-term stability due to the MOSFET (electronic part) protected from the potentially harmful ionic solution while the extended gate (the sensitive part) which is located in the solution is easy to replace. ${ }^{1,9}$

However, boththe ISFET and EGEFET structures need reference electrodes in order to provide a defined reference potential., ${ }^{3,10}$ Unfortunately, commercial reference electrodes are fragile, bulky, expensive, and too large for small-volume solution applications. ${ }^{11}$ In recent years, many attempts have been investigated to replace the commercial 
reference electrodes, for example, by a solid-state planar on-chip reference electrode or a reference field-effect transistor. ${ }^{11,12}$ However, despite these efforts, these methods suffer from poor performance, instability, short life time, and complex fabrication and integration with other microfabrication processes. ${ }^{11-13}$ On the other hand, chemical sensors with interdigitated electrodes (IDEs) have significant advantages over other kinds of arrangements of electrodes: first, the possibility of easy labon-a-chip fabrication and integration, ${ }^{14}$ second, the ability to monitor the resistivity and the permittivity of a solution ${ }^{15}$ third, they have a shallow electric field penetration depth, which makes them sensitive to changes occurring close to their surface, and allows the use of thin sensing films for designing selectivity and sensitivity ${ }^{15}$ and fourth, they offer a large variety of sizes, shapes and materials to address the particular requirements. However, to the best of our knowledge, no papers have reported the use of the interdigitated structure for EGFET despite the appealing properties.

Metal-oxide semiconductor thin films have been widely utilized as sensing materials for the FET-pH sensor membranes. ${ }^{16}$ Among them, zinc oxide ( $\mathrm{ZnO}$ ) thin films have attracted considerable attention due to their unique properties with respect to $\mathrm{pH}$ sensing. ${ }^{17} \mathrm{ZnO}$ is a bio-safe material with a large bandgap energy, electrochemical activity, high electron mobility, high mechanical strength and good chemical and thermal stability, abundant source material, and low fabrication costs. ${ }^{18-21} \mathrm{ZnO}$ thin film-based FET-pH sensor membranes have already been recently reported by many research groups. ${ }^{22-25}$

The challenge here is to obtain acceptable signal quality and linear response for a new EGFET structure without reference electrodes, namely, an interdigitated extended gate field effect transistor (IEGFET). The proposed IEGFET consists of two parts: an interdigitated metal-semiconductor-metal (MSM) extended gate with asymmetric metal contact fingers based on $\mathrm{ZnO}$ thin film as the sensing part, while a commercial MOSFET (model: CD4007UB; RCA Solid State Division) was used as FET to amplify the signal and observe the detection through the modulation of the drain current. This combination of the ubiquitous MOSFET technology with an interdigitated electrode sensor results in sensors that are much cheaper than the alternatives (EGFETs and ISFETs), and much more easily integrated with necessary external electronics. Further, IEGFETs offer the possibility of simple and easy lab-on-a-chip integration for applications for small-volume solutions by eliminating the reference electrode.

\section{MATERIALS AND METHODS}

$\mathrm{ZnO}$ thin film layers were prepared on $p$-type $\mathrm{Si}<100>$ substrates by using the sol-gel method. Prior to the $\mathrm{ZnO}$ films deposition, the substrates were cleaned. The procedure of the cleaning process and $\mathrm{ZnO}$ synthesis are described in detail in our previous works. $^{21,26}$

The $\mathrm{ZnO}$ thin film was spin-coated on the $\mathrm{Si}$ substrate at a speed of $4000 \mathrm{rpm}$ for $40 \mathrm{~s}$. Then, the deposited coatings were preheated on a hot plate at $100^{\circ} \mathrm{C}$ for $5 \mathrm{~min}$ and further post-heated at $450^{\circ} \mathrm{C}$ for $1 \mathrm{~h}$ in a nitrogen atmosphere tube furnace. Planer-type interdigitated MSM electrodes were fabricated on the top surface of the $\mathrm{ZnO}$ films. Ni electrodes were deposited through the shadow mask in a conventional vacuum thermal evaporator system. Ni powder of $99.99 \%$ purity was evaporated from a molybdenum boat. Prior to the deposition, the vacuum chamber was evacuated to a pressure of $10^{-5}$ mbar. The Ni electrodes were deposited up to $200 \mathrm{~nm}$ in thickness at a deposition rate of $0.1 \mathrm{~nm} / \mathrm{s}$.

The finger width, length and spacing of the fabricated devices were $150 \mu \mathrm{m}, 4000 \mu \mathrm{m}$ and $150 \mu \mathrm{m}$, respectively. Figure $1 \mathrm{a}$ and $\mathrm{b}$ shows a schematic diagram of interdigitated MSM extended gate geometry and a cross-section of the interdigitated extended gate.

The characterization of $\mathrm{ZnO}$ thin films via the sol-gel method has been investigated in our previous work. ${ }^{26}$ The details of the characteristics of undoped $\mathrm{ZnO}$ thin film are listed in Table I.

The current-voltage (I-V) characteristics of the IEGFET in the $\mathrm{pH}=4,5,6,7,8,9,10,11$ buffer solution were measured using a Keithley Semiconductor Characterization System (SCS-4200). The measurement setup is shown in Fig. 1c. The twoterminal sensor was dipped in a beaker containing an electrolyte buffer solution for $15 \mathrm{~s}$, The first terminal was connected to the SCS-4200, providing a bias voltage, while the second one was connected to the gate terminal of $n$-MOSFET. A commercial MOSFET (model: CD4007UB; RCA Solid State Division) was used as FET in the IEGFET device. This device has good characteristics and is also commercially available. The MOSFET transistor was connected to the SCS 4200, Keithley, via a bread board. Between each test, the sensing gate was rinsed with DI water for $15 \mathrm{~s}$ in order to remove any residual chemical compounds. All measurements were recorded at room temperature.

\section{RESULTS AND DISCUSSION}

Two techniques have been carried out for estimating the $\mathrm{pH}$ sensor performance ofthe $\mathrm{ZnO}$ IEGFET without a reference electrode. The first one is the constant current technique, and the second is the constant voltage technique. The channel current can be modified using a concentration of $\mathrm{H}^{+}$ions, while the drain-source voltage $V_{\mathrm{DS}}$ is kept constant. Figure 2 shows the transfer characteristics (drain current versus reference electrode voltage, $I_{\mathrm{DS}}-V_{\text {ref }}$ ) in the linear region for the IEGFET sensor for $V_{\mathrm{DS}}$ fixed at $0.2 \mathrm{~V}$ and $V_{\text {ref }}$ varied from 0 to $4 \mathrm{~V}$. According to the EGFET 
(a)

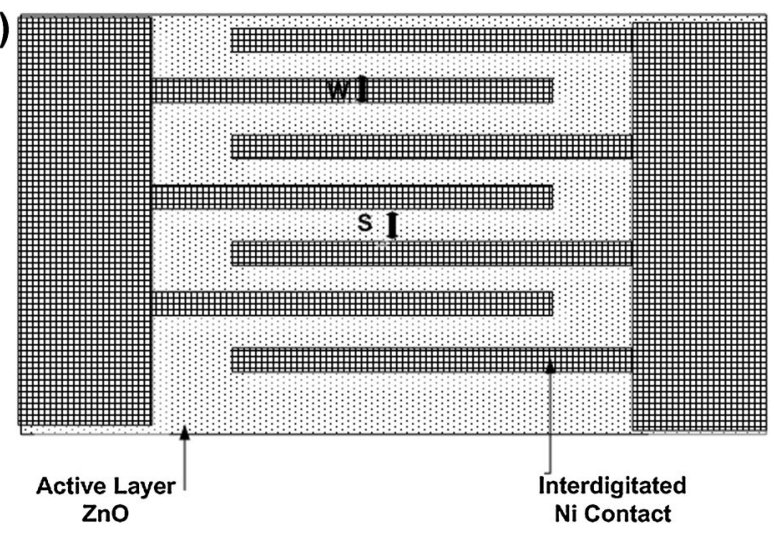

(b)

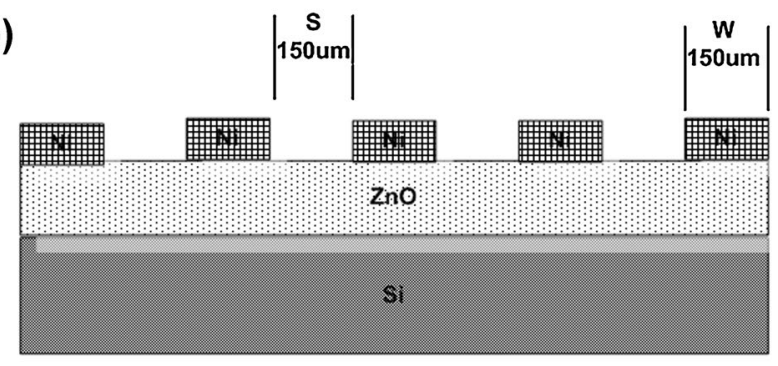

(c)

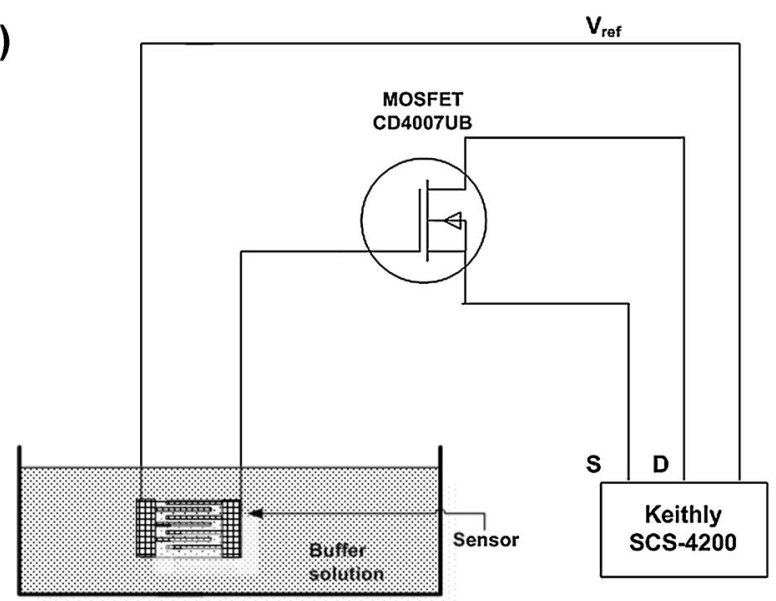

Fig. 1. (a) Interdigitated MSM extended gate with asymmetric metal contact fingers; (b) cross-section of the interdigitated extended gate; and (c) the measurement setup.

Table I. Characteristics of $\mathrm{ZnO}$ thin film

\begin{tabular}{lc}
\hline Parameter & Values \\
Roughness $(\mathrm{nm})$ & 5 \\
Grain size $(\mathrm{nm})$ & 97 \\
Film thickness $(\mathrm{nm})$ & 200 \\
Resistivity $(\Omega \mathrm{m})$ & 32.92 \\
Mobility $\left(\mathrm{cm}^{2} \mathrm{~V}^{-1} \mathrm{~s}^{-1}\right)$ & 21.42 \\
Carrier concentration $\left(\mathrm{cm}^{-3}\right)$ & $9.23 \times 10^{15}$ \\
Band gap $(\mathrm{eV})$ & 3.41 \\
\hline
\end{tabular}

theory, the $V_{\mathrm{T}(\mathrm{IEGFET})}$ could be estimated by using the relationship ${ }^{5,6}$ :

$$
V_{\mathrm{T}(\mathrm{IEGFET})}=V_{\mathrm{T}(\mathrm{MOSFET})}-\frac{\varphi_{M}}{q}+E_{\mathrm{ref}}+\chi^{\mathrm{sol}}-\psi
$$

where $V_{\mathrm{T}(\mathrm{MOSFET})}$ is the MOSFET threshold voltage, $\Phi_{\mathrm{M}}$ is the metal gate work function, $E_{\mathrm{ref}}$ is the reference electrode potential, $\chi^{\text {sol }}$ is the buffer solution surface dipole potential and $\psi$ is the surface potential at the solution/sensing film interface.

$V_{\text {T(IEGFET) }}$ exhibits $\mathrm{pH}$-dependence, since the surface potential, $\psi$, on the $\mathrm{ZnO} / \mathrm{Ni}$ gate varies with $\mathrm{pH}$ in different solutions. The operation of the IEGFET is very similar to the conventional MOSFET except that the metal gate is replaced by the interdigitated structure on the sensing film, i.e., the IEGFET is based on the formation of a double layer at the solution/oxide film interface that generates a surface potential which changes the turn-on voltage, $V_{\text {T(IEGFET), enabling the ion concentration in a }}$ solution to be determined. $6,27,28$

It is seen in Fig. 2a that the $I_{\mathrm{DS}}-V_{\mathrm{REF}}$ curves in the linear region show a shift from left to right of the $\mathrm{V}_{\text {T(IEGFET) }}$ with increasing the $\mathrm{pH}$ value. The positive shifting in the $V_{\text {T(IEGFET) }}$ is due to the decreasing of the $\psi$, according to Eq. 1 . This decreasing in the $\psi$ can be attributed to the decreasing in amount of $\mathrm{H}^{+}$ions accumulated onto the $\mathrm{ZnO}$ surface. ${ }^{29}$ Decreasing in $\mathrm{H}^{+}$ions leads to increasing of the $\mathrm{pH}$ values of the buffer solution, according to the definition of $\mathrm{pH}$.

The $\mathrm{pH}$ voltage sensitivity of the $\mathrm{ZnO}$ IEGFET was extracted from the $I_{\mathrm{DS}}-V_{\mathrm{REF}}$ curves at $I_{\mathrm{DS}}=200 \mu \mathrm{A}$ in the $\mathrm{pH}$ value of the buffer solution ranging from $\mathrm{pH}$ 4 to $\mathrm{pH} 11$. The $\mathrm{pH}$ voltage sensitivity curve (reference voltage versus $\mathrm{pH}$ values) of the $\mathrm{ZnO}$ sensing film is shown in Fig. 2b. This sensitivity can be derived by linear fitting as follows;

$$
p H_{-} \text {voltage_seneitivity }=\frac{\Delta V_{T}}{\Delta p H}=\frac{\Delta V_{\text {ref }}}{\Delta p H}
$$

The sensitivity and linearity of the $\mathrm{ZnO}$ IEGFET sensor without reference electrode were found to be $22.4 \mathrm{mV} / \mathrm{pH}$ and $92.8 \%$, respectively. To compare and contrast the performance of the IEGFET with conventional EGFET configurations, the measurements were also performed by using a reference electrode with the studied interdigitated extended gate as a conventional one. The measurement setup is described in our previously reported work for a conventional structure. ${ }^{30}$ However, the conventional configuration shows a slightly higher performance. The voltage sensitivity and linearity are found to be $26.5 \mathrm{mV} / \mathrm{pH}$ and $94.3 \%$, respectively. Generally speaking, the voltage sensitivity and linearity in our ZnO IEGFET sensor is slightly lower than those previously reported for a conventional devices of ZnO EGFET. ${ }^{19,20,22,23,30,31}$ Regardless of IEGFET has lower voltage sensitivity and 
linearity than EGFET, the key point is that the proposed device does not need a reference electrode component and shows acceptable voltage sensitivity and linearity. The improvement of the proposal structure performance is another issue, which could be achieved by studied different materials for sensing membranes (e.g. polymers, other semiconductor oxide), structure of sensing membranes (e.g.
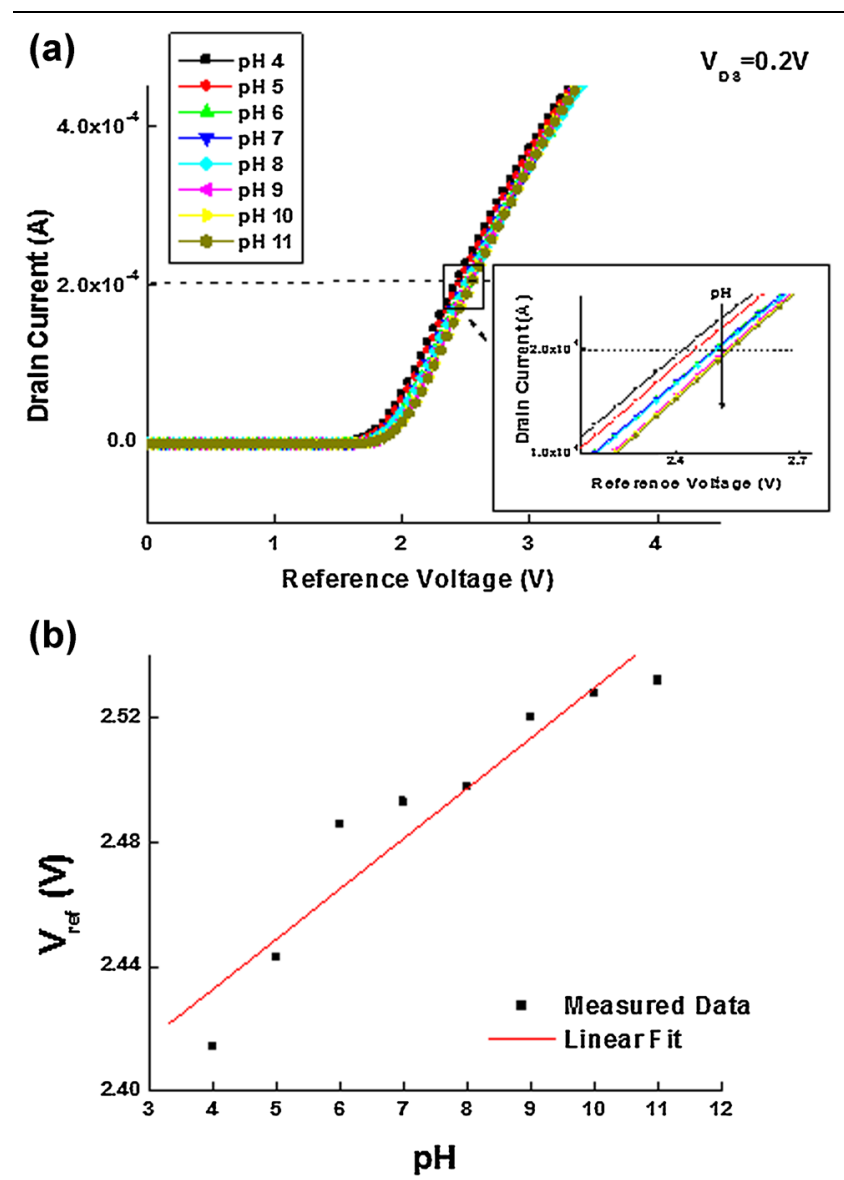

Fig. 2. (a) Drain current versus reference voltage measurements in $\mathrm{pH}=4$ to 11 buffer solutions. Inset the change magnified at 2.2-2.7 V. (b) Reference voltage versus $\mathrm{pH}$ values of the $\mathrm{ZnO}$ thin film IEGFET sensors. nanostructure thin film), and the size and shape of interdigitated extended gate structures etc. Table II summarizes the performance of the proposed undoped $\mathrm{ZnO}$ IEGFET $\mathrm{pH}$ sensor with the previously reported undoped $\mathrm{ZnO}$ EGFET $\mathrm{pH}$ sensors.

The second mode to estimate the IEGFET $\mathrm{pH}$ sensor is the constant voltage technique. At the gate-source voltage $\left(V_{\mathrm{GS}}\right)$ equal to $3 \mathrm{~V}, V_{\mathrm{DS}}$ was varied from $0 \mathrm{~V}$ to $5 \mathrm{~V}$ by $0.1 \mathrm{~V} / \mathrm{step}$, and the drain current versus drain source voltage $\left(I_{\mathrm{DS}}-V_{\mathrm{DS}}\right)$ curves for varying $\mathrm{pH}$ values for the IEGFET $\mathrm{pH}$ sensor were measured. The obtained results shown in Fig. 3a demonstrate that the drain-source current downward shifts to a lower value relative to an increase in $\mathrm{pH}$ values.

According to the ISFET and EGFET theories, the IV characteristics in the saturation region can be described as follows ${ }^{6}$

$$
I_{\mathrm{DS}}=\frac{w \mu_{n} C_{o x}}{2 l}\left(V_{r e f}-V_{\mathrm{T}(\mathrm{IEGFET})}\right)^{2}
$$

where $w$ is the channel width, $l$ is the channel length ratio, $\mu_{\mathrm{n}}$ is the channel electron mobility, $C_{\mathrm{ox}}$ is the gate capacitance per unit area, $V_{\mathrm{T} \text { (IEGFET) }}$ is the threshold voltage of the IEGFET device and $V_{\text {ref }}$ is the reference voltage. The downward shifting in the drain-source current $\left(I_{\mathrm{DS}}\right)$ upon increasing the $\mathrm{pH}$ value is due to the increasing of the $V_{\text {T(IEGFET) }}$, as shown in Eq. 3 and Fig. 3a. The pH current sensitivity and linearity of the ZnO IEGFET was extracted from the $I_{\mathrm{DS}}-V_{\mathrm{DS}}$ curves at $V_{\mathrm{DS}}=2.5 \mathrm{~V}$ at a $\mathrm{pH}$ value of the buffer solution ranging from $\mathrm{pH} 4$ to $\mathrm{pH}$ 11. Current sensitivity curves ( $\mathrm{I}_{\mathrm{DS}}$ vs. $\mathrm{pH}$ values) of the $\mathrm{ZnO}$ IEGFET sensor are shown in Fig. 3b. The $\mathrm{pH}$ voltage sensitivity can be derived by linear fitting as follows

$$
p H \_ \text {current_seneitivity }=\frac{\Delta I_{D S}}{\Delta p H}
$$

The $\mathrm{pH}$ current sensitivity was found to be about 45 $\mu \mathrm{A} / \mathrm{pH}$ with a linearity of $96.2 \%$ which are both in agreement with the values previously reported for a conventional ZnO EGFET. ${ }^{20,30}$

Table II. Comparison of voltage sensitivities of the proposed ZnO IEGFET and conventional ZnO EGFET pH

\begin{tabular}{|c|c|c|c|}
\hline Reference & Voltage sensitivity $(\mathrm{mV} / \mathrm{pH})$ & Linearity & PH range \\
\hline (This work proposed IEGFET) & 22.4 & 0.928 & $4-11$ \\
\hline (This work conventional EGFET) & 26.5 & 0.943 & $4-11$ \\
\hline Ref. 19 & 35.32 & 0.9757 & $1-13$ \\
\hline Ref. 20 & 46.25 & 0.99 & $1-13$ \\
\hline Ref. 22 & 38 & NA & $2-12$ \\
\hline Ref. 23 & 38.46 & NA & $4-12$ \\
\hline Ref. 30 & 24.41 & 97.7 & $5-11$ \\
\hline Ref. 31 & 38.2 & NA & $4-10$ \\
\hline
\end{tabular}
sensors

NA Not available. 

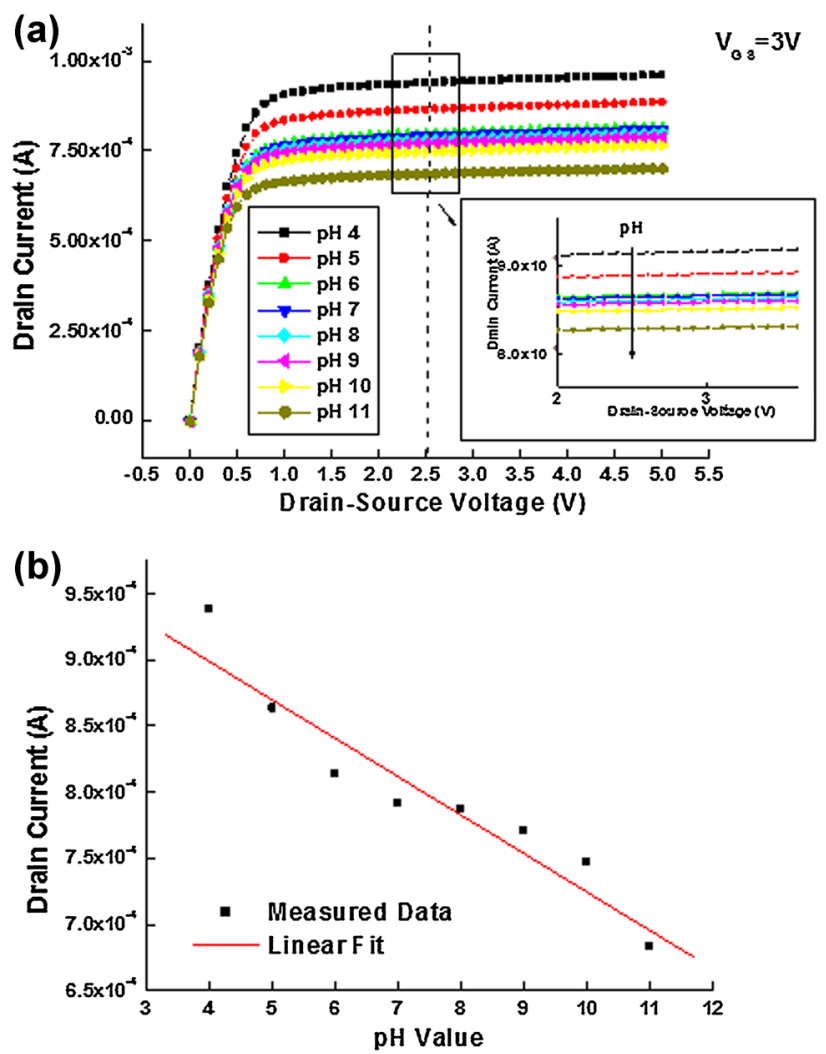

Fig. 3. (a) Drain current versus drain-source voltage measurements in $\mathrm{pH}=4-11$ buffer solutions. Inset shows the change magnified at 2-3.6 V. (b) Drain current versus $\mathrm{pH}$ values of the $\mathrm{ZnO}$ thin film IEGFET sensors.

\section{CONCLUSIONS}

We have investigated here for the first time an IEGFET device without a reference electrode, which can be used as a $\mathrm{pH}$ sensor. The sensing structure consists of IDEs based on $\mathrm{ZnO}$ thin film as an extended gate. The proposed IEGFET could easily be extended to other types of sensors. The device has real-time and linear $\mathrm{pH}$ voltage and current sensitivities in a $\mathrm{pH}$ concentration ranging from $\mathrm{pH}$ 4 to $\mathrm{pH}$ 11. The IEGFET $\mathrm{pH}$ current sensitivity is found to be about $45 \mu \mathrm{A} / \mathrm{pH}$ which is agreement with the conventional configuration of $\mathrm{ZnO}$ EGFETs. However, the voltage sensitivity at $22.4 \mathrm{mV} / \mathrm{pH}$ is lower than those previously reported for a conventional EGFET. The proposed IEGFET performance could be enhanced by modifying the size, shape and material type and structure of the interdigitated extended gate. The measurement by IEGFET is very simple and needs just a small volume of solution to test, which gives opportunities for miniaturization and lower manufacturing costs.

\section{REFERENCES}

1. J.C. Fernandes, R. Nascimento, and M. Mulato, Mater. Res. 19, 1 (2016).

2. D.-S. Kim, J.-E. Park, J.-K. Shin, P.K. Kim, G. Lim, and S. Shojii, Sens. Actuators B Chem. 117, 488 (2006).

3. P. Bergveld, IEEE Sensor Conference, Toronto (2003), pp. $1-26$.

4. E.M. Guerra, G.R. Silva, and M. Mulato, Solid State Sci. 11,456 (2009).

5. K. Wang, W. Tsai, P. Yang, C. Chou, Y. Li, C. Liao, and H. Cheng, Jpn. J. Appl. Phys. 54, 04DL01 (2015).

6. A. Das, D.H. Ko, C.-H. Chen, L.-B. Chang, C.-S. Lai, F.-C. Chu, L. Chow, and R.-M. Lin, Sens. Actuators B Chem. 205, 199 (2014).

7. N. Al-Hardan, M.A. Hamid, N. Ahmed, A. Jalar, R. Shamsudin, N. Othman, L.K. Keng, W. Chiu, and H. Al-Rawi, Sensors 16, 839 (2016).

8. J. Van Der Spiegel, I. Lauks, P. Chan, and D. Babic, Sens. Actuators 4, 291 (1983).

9. L.L. Chi, J.C. Chou, W.Y. Chung, T.P. Sun, and S.K. Hsiung, Mater. Chem. Phys. 63, 19 (2000).

10. M.H. Asif, O. Nur, M. Willander, and B. Danielsson, Biosens. Bioelectron. 24, 3379 (2009).

11. C. Duarte-Guevara, V.V. Swaminathan, M. Burgess, B. Reddy, E. Salm, Y.-S. Liu, J. Rodriguez-Lopez, and R. Bashir, Analyst 140, 3630 (2015).

12. M. Myers, F.L.M. Khir, A. Podolska, G.A. Umana-Membreno, B. Nener, M. Baker, and G. Parish, Sens. Actuators B Chem. 181, 301 (2013).

13. M. Waleed Shinwari, D. Zhitomirsky, I.A. Deen, P.R. Selvaganapathy, M. Jamal Deen, and D. Landheer, Sensors 10,1679 (2010).

14. L.H.D. Skjolding, C. Spegel, A. Ribayrol, J. Emnéus, and L. Montelius, J. Phys. Conf. Ser. 100, 052045 (2008).

15. R. de la Rica, C. Fernández-Sánchez, and A. Baldi, Electrochem. Commun. 8, 1239 (2006).

16. P. Kurzweil, Sensors 9, 4955 (2009).

17. S. Al-Hilli and M. Willander, Sensors 9, 7445 (2009).

18. C.T. Lee, Y.S. Chiu, S.C. Ho, and Y.J. Lee, Sensors 11, 4648 (2011).

19. J.-L. Wang, P.-Y. Yang, T.-Y. Hsieh, C.C. Hwang, and M.-H. Juang, J. Nanomater. 2013, 152079 (2013).

20. H.H. Li, C.E. Yang, C.C. Kei, C.Y. Su, W.S. Dai, J.K. Tseng, P.Y. Yang, J.C. Chou, and H.C. Cheng, Thin Solid Films 529, 173 (2013).

21. G.M. Ali, C.V. Thompson, A.K. Jasim, I.M. Abdulbaki, and J.C. Moore, Sensors 13, 16801 (2013).

22. P.D. Batista and M. Mulato, Appl. Phys. Lett. 87, 1 (2005).

23. Y.S. Chiu, C.Y. Tseng, and C.T. Lee, IEEE Sens. J. 12, 930 (2012).

24. P.Y. Yang, J.L. Wang, P.C. Chiu, J.C. Chou, C.W. Chen, H.H. Li, and H.C. Cheng, IEEE Electron Device Lett. 32, 1603 (2011)

25. H.J. Jang, J.G. Gu, and W.J. Cho, Sens. Actuators B Chem. 181, 880 (2013).

26. G.M. Ali, J.C. Moore, A.K. Kadhim, and C. Thompson, Sens. Actuators A Phys. 209, 16 (2014).

27. C.S. Lee, S. Kyu Kim, and M. Kim, Sensors 9, 7111 (2009).

28. P.C. Yao, J.L. Chiang, and M.C. Lee, Solid State Sci. 28, 47 (2014).

29. Y.S. Chien, W.L. Tsai, I.C. Lee, J.C. Chou, and H.C. Cheng, IEEE Electron Device Lett. 33, 1622 (2012).

30. G. M. Ali, R. H. Dhaher, A. A. Abdullateef, Third International Conference Technological Advances in Electrical Electronics, Computer Engineering, Beirut (2015), pp. 234238.

31. B. Rosli, N. D. H. Abd Patah, M. Rosdan, M. M. Marbie, M. H. Juhari, S. S. Shariffudin, S. H. Herman, and M. Rusop, RSM 2013 IEEE Regional Symposium Micro Nano Electronics (2013), p. 171. 